\title{
Self-control and health-related behaviour: The role of implicit self-control, trait self-control, and lay beliefs in self-control
}

\author{
Martin S. Hagger ${ }^{1,2,3,4 *}$ (D), Daniel F. Gucciardi ${ }^{5}$ (D), Amelia S. Turrell ${ }^{4}$ \\ and Kyra Hamilton ${ }^{3,4}$
}

'Psychological Sciences and Health Sciences Research Institute, University of California, Merced, California, USA

${ }^{2}$ Faculty of Sport and Health Sciences, University of Jyvaskyla, Jyvaskyla, Finland

${ }^{3}$ School of Applied Psychology, Menzies Health Institute Queensland, Griffith University, Mt. Gravatt, Queensland, Australia

${ }^{4}$ School of Psychology, Curtin University, Perth, Western Australia, Australia ${ }^{5}$ School of Physiotherapy and Exercise Science, Curtin University, Perth, Western Australia, Australia

Objectives. We tested unique contribution of trait self-control, implicit self-control, and lay beliefs in self-control beliefs to the prediction of health-related behaviours. We also tested whether relations between trait self-control and health-related behaviour, and between implicit self-control and health-related behaviours, were moderated by selfcontrol beliefs.

Design. Cross-sectional, correlational.

Methods. Students $(N=176)$ completed self-report measures of trait self-control, lay beliefs that self-control is limited or non-limited, non-planning, participation in healthrelated behaviours (impulsive eating, impulsive drinking, exercise avoidance, watching diet, alcohol consumption, physical activity participation), and demographic variables. Participants also completed a measure of implicit self-control using an implicit association test.

Results. Analyses indicated significant negative relations between implicit self-control and impulsive drinking and alcohol consumption. We also found a positive relationship between implicit self-control and exercise behaviour, and a negative relationship between implicit self-control and impulsive eating, both of which fell marginally short of statistical significance. Trait self-control significantly predicted all behavioural measures and attenuated relations between implicit self-control and health-related behaviour. We found no relations between lay beliefs in self-control and health-related behaviour. Moderated path analyses revealed that lay beliefs in self-control moderated relations between trait self-control and impulsive drinking, implicit self-control and exercise avoidance, and implicit self-control and physical activity participation.

Conclusions. Findings suggest that trait self-control was a consistent correlate of health-related behaviour, while the effect sizes of relationships between implicit self-

* Correspondence should be addressed to Martin S. Hagger, Psychological Sciences and Health Sciences Research Institute, University of California, Merced, 5200 N. Lake Road, Merced, CA 95343, USA (email: mhagger@ucmerced.edu). 
control and health-related behaviours were small. Strength of relations between trait selfcontrol and health-related behaviours may depend on whether or not individuals believe self-control is limited.

\section{Statement of contribution}

\section{What is already known on this subject?}

- Self-control represents individuals capacity to override impulses, break habits, and avoid temptations.

- Trait self-control is consistently and positively related to uptake of health-promoting behaviours (e.g., physical activity, healthy eating).

- Trait self-control is consistently and negatively related to health-compromising behaviours (e.g., alcohol consumption, unhealthy snacking).

\section{What does this study add?}

- Proposes that self-control can also be measured implicitly, beyond individuals' awareness.

- Examined effects of implicit measures of self-control and self-reported trait self-control on multiple health behaviours.

- Effects of implicit self-control on health behaviours were small and subsumed by trait self-control.

- Effects of lay beliefs in self-control moderated effects of implicit and trait self-control on some health behaviours.

Epidemiological research has identified the behavioural origins of chronic non-communicable diseases and conditions such as cardiovascular disease, some cancers, and diabetes (Ford, Zhao, Tsai, \& Li, 2011). As a consequence, government health departments and health advocacy organizations have called for the development of behavioural interventions to curb the insidious human and economic costs of chronic disease (OSBBR, 2016). Behavioural interventions targeting change in behaviour at the individual level necessitate the identification of the key modifiable determinants of healthpromoting behaviours (Sheeran, Klein, \& Rothman, 2017). Identification of the determinants is necessary to flag the key targets for behavioural interventions and the development of interventions with the highest probability of successful health behaviour change (Kok et al., 2016).

For most people, participating in health-promoting behaviours requires considerable effort and investment of cognitive resources (Baumeister \& Heatherton, 1996; Hofmann, Schmeichel, \& Baddeley, 2012). People have to forego or suppress the 'default' behavioural option, which is often the one that has been the most frequently performed in the past, highly rewarding, reinforced behaviourally and socially, and enacted impulsively with little thought or effort, in favour of a more effortful, cognitively demanding behavioural option. For example, for individuals to opt to introduce healthy meal choices into their diet, they have to forgo the highly rewarding palatable meals that they routinely eat. Participation in health behaviours is, therefore, highly dependent on individuals' capacity to override the reinforcing contingencies that cue up the default, unhealthy options (De Ridder \& De Wit, 2006; Hagger, 2014). In addition, maintaining health behaviours is dependent on the individual's capacity to engage in the more effortful behavioural option for a sustained period (Duckworth \& Gross, 2014).

Self-control is a key individual difference construct that has been consistently linked with individuals' capacity to override impulsive, immediately rewarding behaviours and engage in sustained, effortful goal-oriented action (Allom et al., 2018; de Ridder, LensveltMulders, Finkenauer, Stok, \& Baumeister, 2012; Tangney, Baumeister, \& Boone, 2004). 
Trait self-control has frequently been assessed using self-report instruments (e.g., Grasmick, Tittle, Bursik, \& Arneklev, 1993; Tangney et al., 2004). Primary research (Hamilton, Fleig, \& Hagger, 2019; Hankonen, Kinnunen, Absetz, \& Jallinoja, 2014; Junger \& van Kampen, 2010; Tangney et al., 2004) and meta-analytic evidence (de Ridder et al., 2012) indicate that trait measures of self-control are positively associated with participation in health-promoting behaviours such as healthy eating and weight control, and with adaptive outcomes such as interpersonal adjustment and well-being and adjustment.

Researchers interested in the mechanisms underpinning relations between trait selfcontrol and behaviour have identified two prominent pathways: a deliberative process in which trait self-control confers increased motivation to engage in goal-directed behaviour, and greater capacity to actively monitor and resolve cues to impulsive behaviours, and an implicit process in which individuals are biased towards control-related cues and away from cues to impulse-related behaviours. Theory on self-control suggests that trait selfcontrol reflects individuals' capacity for impulse suppression and regulation of action over time (Paschke et al., 2016), and their ability to monitor and attend to cues to engage in goal-directed behaviours, and disregard or manage cues for behaviours that may derail the goal-directed actions (Baldwin, Finley, Garrison, Crowell, \& Schmeichel, 2018; Gottfredson \& Hirschi, 1990). The reported relations between self-report measures of trait selfcontrol and health-related outcomes may reflect both sets of processes. However, to date, there has been little research examining self-control implicitly, and the extent to which it may account for relations between trait self-control on behaviour.

Measures of implicit self-control may provide a means to establish the extent to which relations between trait self-control and behaviour are attributable to implicit biases, and whether such biases affect behaviours independently. Research examining relations between trait self-control and health behaviours, for example, has identified indirect effects on behavioural outcomes mediated by social cognition and intentions, as well as direct effects (Hankonen et al., 2014). The indirect effects likely represent the deliberate, reasoned process by which trait self-control affects behaviour. For example, individuals' trait self-control serves as a source of information for individuals forming beliefs and intentions to perform the behaviour in future, similar to previous research that has identified indirect effects of traits related to motivation and effort (e.g., self-discipline, conscientiousness) on health behaviour (e.g., Conner \& Abraham, 2001; Courneya, Bobick, \& Schinke, 1999). Residual effects of trait self-control on behaviour, however, likely reflect an implicit process by which trait self-control impacts behaviour (Hagger, 2013). Such a pathway may indicate that trait self-control affords individuals the capacity to resist impulses and manage cues that derail goal-directed behaviour beyond their awareness. The availability of reaction time measures of individual difference constructs, such as the implicit association test (IAT; Greenwald, McGhee, \& Schwartz, 1998), provides the opportunity to measure implicit self-control and test the independent relations between implicit self-control and health-related behaviours alongside trait selfcontrol. Such research would provide important data on the extent to which relations between self-control and behaviour may reflect an implicit, non-conscious process.

\section{Lay beliefs in self-control}

A recent development in the self-control literature has been to examine the role that individuals' implicit theories about self-control have in determining their self-control capacity and subsequent behaviour (Bernecker \& Job, 2015; Job, Dweck, \& Walton, 2010; 
Job, Walton, Bernecker, \& Dweck, 2015). Researchers have demonstrated that individuals who believe that self-control is not a fixed or limited capacity, and can be incrementally improved or applied flexibly, have better self-control capacity, and appear not to suffer as greatly from the deleterious effects of availability of self-control resources (Job et al., 2010), and are also more effective in goal enactment and sustained self-regulation (Job et al., 2015). However, there is relatively little research assessing the extent to which such beliefs affect behaviour independent of trait self-control, and such research has the potential to provide important information on the extent to which relations between of trait self-control and behaviour may be attributable to beliefs in whether or not self-control is limited.

We also tested whether or not beliefs in self-control had a role in determining the extent to which trait self-control and implicit self-control relate to behaviour. We propose two competing predictions. One prediction derived from previous research is that a belief that self-control is not limited leads individuals to be more effective in exerting self-control (Job et al., 2010). This belief may translate into larger relations between trait self-control and behaviour when individuals endorse a non-limited model of self-control, and smaller relations among those who view self-control as limited. However, research demonstrating that self-control capacity is moderated by self-control beliefs has tended to focus on state demands on self-control (Job et al., 2010), and previous research examining trait selfcontrol and beliefs in self-control have found independent prediction of behaviour by both types of self-control, but did not test any interaction effect (Job et al., 2015). Given that trait self-control and implicit self-control likely represent relatively fixed, stable conceptualizations of self-control, we propose an alternative hypothesis that the behaviour of individuals who believe that self-control is limited may be more likely to be predicted by trait self-control and implicit self-control. In contrast, the behaviour of individuals who hold beliefs that self-control is non-limited may be less likely to be predicted by trait and implicit self-control. Behaviour for such individuals may, instead, be more subject to influence from situational motivational factors, such as state levels of selfcontrol resources or their self-efficacy towards that particular behaviour in that particular context. For example, among individuals endorsing beliefs that trait self-control is limited, those who have high levels of trait self-control may be more likely to drink less alcohol and do more exercise, but those who have low levels of trait self-control may be more likely to avoid exercise and drink more alcohol. In contrast, individuals that endorse the belief that self-control is non-limited may be less subject to trait self-control in the prediction of their behaviour. Trait self-control may still affect their behaviour and follow a similar pattern to those with beliefs that self-control is limited, but the size of the relations between trait selfcontrol and behaviour may be diminished. Testing whether or not the strength of the relationship between trait self-control and health behaviour, and between implicit selfcontrol and health behaviour, varies according to individuals' beliefs in self-control would provide important information on the conditions under which trait self-control relates to health behaviour.

\section{Aims and hypotheses}

The aim of the present study was to test the relative contribution of trait self-control, implicit self-control, and self-control beliefs to the prediction of health-related behaviours. We aimed to test relation between a measure of implicit self-control and a series of healthrelated behaviours (impulsive eating, impulsive alcohol drinking, and exercise avoidance; watching diet, alcohol consumption, and physical activity participation) alongside effects 
of trait self-control and lay beliefs in self-control. We also aimed to provide concurrent validity for the self-control constructs alongside a measure of non-planning, a key component of the impulsivity. We hypothesized that all three self-control variables would be positively correlated, and negatively correlated, with non-planning. We predicted that implicit self-control would be negatively related to impulsive eating, impulsive drinking, exercise avoidance, and alcohol consumption, and positively related to watching diet and alcohol consumption. We also predicted a similar pattern of relations for trait self-control with negative effects on impulsive eating, impulsive drinking, exercise avoidance, and alcohol consumption, and positive effects on watching diet and alcohol consumption, consistent with previous research (de Ridder et al., 2012). In addition, we hypothesized that the relationship between implicit self-control and health behaviours would be attenuated by trait self-control, and we expected this to be reflected in indirect effects of implicit self-control on behaviour through trait self-control. We expected the attenuation effect to be independent of self-control beliefs, and that self-control beliefs would have unique relations with health behaviours. Finally, we also tested whether or not beliefs in self-control moderated relations between trait self-control and health behaviours, and between implicit self-control and health behaviours. Given that we propose two competing hypotheses regarding these relations, we make no specific prediction as to their direction.

\section{Method}

\section{Participants}

Participants were undergraduate students studying psychology ( $N=176$; male, $n=52$, female, $n=119$, prefer not to say, $n=5$; mean age $=24.20$ years, $S D=6.77$ ) from Curtin University in Perth, Australia and Griffith University in Brisbane, Australia who completed the study for course credit or a coffee voucher. Based on participants' selfreports of their parents' highest attained level of education, $31.82 \%$ of the participants' parents had completed a University education, with the remainder completing high school. Recruitment was undertaken face-to-face or via an online participant pool management system. Participants were invited to participate in a study that aimed to 'explore the relationship between psychological attributes and health-related behaviour among adults'. Participants were allocated to an available timeslot and were asked to attend the laboratory at the allotted time.

\section{Design and procedure}

The current study adopted a cross-sectional correlational design. Participants attended a single laboratory session during which they completed study measures in an online survey and a measure of implicit self-control using an IAT. Each participant was welcomed to the laboratory by the experimenter and shown into an experimental cubicle equipped with a desk and personal computer. The participant then read a study information sheet presented on the computer screen, which provided a brief outline of the study procedure, expectations, and duration. After having the opportunity to ask questions, the participant was asked to complete an online consent form. Next, the participant was provided with instructions presented on the computer screen on how to complete the self-control IAT and the online survey. The experimenter was on hand to assist with launching the IAT and online questionnaire, and left the cubicle, while the participant was completing each measure. On completion of the IAT and questionnaire, the experimenter provided 
participants with a debrief statement, informed them that the experiment was complete, and thanked them for their assistance. The laboratory session typically lasted $40 \mathrm{~min}$. Ethical approval for study procedures was secured in advance from the University IRBs.

\section{Measures}

Participants completed multi-item self-report measures of study constructs based on published guidelines and measures used in previous studies (Caudwell \& Hagger, 2015; Hagger, Chatzisarantis, \& Harris, 2006; Job et al., 2010; Patton, Stanford, \& Barratt, 1995; Tangney et al., 2004) and the self-control IAT developed specifically for the current study. Complete study measures including items, response scales, and sources are provided in Appendix S1.

\section{Trait self-control}

Trait self-control was measured using the brief self-control scale (Hagger et al., 2018; Tangney et al., 2004). The measure comprises 13 items (e.g., 'I am good at resisting temptation') with responses provided on 5 -point scales $(1=$ not at all and 5 = very much). The scale was scored so that high scores represent higher trait selfcontrol.

\section{Self-control beliefs}

Self-control beliefs based on participants' implicit theories were measured using Job et al.'s (2010) implicit theories about willpower scale. Participants responded to 13 items (e.g., 'People have a certain amount of self-control; there isn't much they can do to change that') with responses provided on 6-point scales $(1=$ strongly disagree and $6=$ strongly agree). The scale was scored so that high scores represented beliefs that self-control is non-limited.

\section{Non-planning}

Items from the Barratt impulsiveness scale were included in the survey. We computed the non-planning factor (Patton et al., 1995), which comprises items from the self-control ('I plan tasks carefully', reverse scored) and cognitive complexity ('I like to think about complex problems', reverse scored) sub-scales, a total of ten items. Responses were provided on 4-point scales $(1=$ rarely/never and $4=$ almost always/always $)$. Higher scores on the scale represent greater levels of impulsivity.

\section{Behaviour}

Participants self-reported their general participation in three behaviours related to impulse control: impulsive eating, impulsive alcohol drinking, and exercise avoidance. Participants were presented with an initial instruction: 'Using the scales below, please indicate how often you do the following activities' followed by five items measuring impulsive eating (e.g., 'snacking on junk food'), six items measuring impulsive drinking (e.g., 'getting drunk'), and five items measuring exercise avoidance (e.g., 'avoiding physical exercise'), with responses provided on 5 -point scales $(1=$ never and $5=$ all of the time). Participants also self-reported their recent participation in three health-related behaviours based on measures from previous studies (Caudwell \& Hagger, 2015; Hagger 
et al., 2006): alcohol consumption, watching diet, and vigorous physical activity. Weekly alcohol consumption over the previous 4 weeks was reported on four items, one for each week (e.g., 'How many units (standard drinks) did you consume last week?'), with responses provided in open-ended response boxes. Watching diet over the past 2 weeks was measured on two items (e.g., 'In the course of the past 2 weeks, how often have you watched your diet?'), with responses provided on 6-point scales $(1=$ never and $6=$ every day). Participation in vigorous physical activity over the previous 2 weeks was measured on two items (e.g., 'In the course of the past 2 weeks, how often have you participated in vigorous physical activity for $20 \mathrm{~min}$ at a time?'), with responses provided on 6-point scales $(1=$ never and $6=$ every day $)$.

\section{Demographic variables}

Participants self-reported their gender (coded as $1=$ male, $2=$ female), age in years, and their parents' highest attained education level (coded as $1=$ completed tertiary education, 2 = completed up to secondary/high school education).

\section{Implicit association test}

Implicit self-control was measured using an IAT based Greenwald et al.'s (1998) original measure. The self-control IAT was developed from first principles with item stimuli identified from a review of self-control definitions and measures, and an open-ended questionnaire administered to a pilot sample. Items were selected based on a pilot study (see Appendix S2 for full details) and incorporated into an existing IAT script using the Eprime experimental software. The IAT comprised five blocks of trials with each trial representing a single presentation of a stimulus item. Blocks 1, 2, and 4 were practice blocks in which participants familiarized themselves with the key press corresponding to the target concept categories (Block 1), attribute categories (Block 2), and the target concept categories reversed (Block 4), with each block comprising 20 trials presented in random order. Blocks 3 and 5 were the test blocks comprising 40 trials each. In the test blocks, each trial comprised a stimulus item from the target concept categories (selfcontrol and impulsivity) presented in the centre of the screen and participants were required to match the item with the appropriate self or other attribute category printed in the upper corners of the screen via a left or right key press (see Appendix S3 for an illustration, supplemental materials). Latencies and accuracy of participants' key press in response to each item were logged by the computer. Averaged response latencies for items in blocks 3 and 5 were used to compute the $D$ measure of implicit self-control using Greenwald, Nosek, and Banaji's (2003) scoring algorithm, with larger scores representing higher implicit self-control.

\section{Data analysis}

We tested our hypotheses using a three-step path analytic approach with a robust maximum likelihood estimator implemented using the lavaan package (Rosseel, 2012) in R (R Development Core Team, 2017) ${ }^{1}$. We tested our initial hypothesis that implicit selfcontrol would predict the health-related behaviours by specifying a simple model (Model

\footnotetext{
${ }^{\prime}$ Data files, analysis scripts and supplemental materials are available online: https://osf.io/ywrzc
} 
Martin S. Hagger et al.

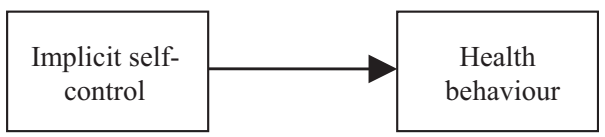

Figure I. Path model depicting regression of health behaviour on implicit self-control (Model I).

1, Figure 1) in which each behavioural measure was regressed on the $D$-score of the implicit self-control IAT. We tested whether implicit self-control accounted for unique variance in each behaviour by specifying a model (Model 2, Figure 2) in which each behavioural measure was regressed on implicit self-control along with measures of trait self-control and self-control beliefs. Finally, in order to test whether any reductions in the unique relation between implicit self-control and each behaviour could be attributed to trait self-control, we estimated a model (Model 3, Figure 3), which was identical to Model 2 with the exception of the specification of a direct relationship between implicit selfcontrol and trait self-control ${ }^{2}$. This enabled the computation of an indirect effect of implicit trait self-control on behaviour through trait self-control, which provides an indication of the extent to which variance shared by implicit self-control and behaviour is accounted for by trait self-control. In addition, we computed the effect size of the indirect effect using Lachowicz, Preacher, and Kelley (2018) upsilon measure. We controlled for gender, age, and parents' highest education level in each model by including them as independent predictors of behaviour. The independent variables in each model were intercorrelated, as is convention in path analyses. A statistical power analysis using $G^{*}$ Power indicated that a sample size of 139 was necessary to detect a small-to-medium effect size, $d=.26$, consistent with previous meta-analytic effects of trait self-control of health behaviour (de Ridder et al., 2012). The analysis was based on a regression model with six predictors (trait self-control, implicit self-control, self-control beliefs, age, gender, and parents' highest education level) with power set at .90 and alpha set at $.01 .^{3}$

We tested our hypothesis that beliefs in self-control would moderate the relationship between trait self-control and health-related behaviour, and between implicit self-control and health-related behaviour, using moderated regression (Figure 4, Model 4). Specifically, each behaviour was regressed on mean-centred trait self-control or implicit selfcontrol and beliefs in self-control variables, and the multiplicative composite of the two centred variables to model the interaction effect. Models were estimated with bootstrapped standard errors with 1000 replications (Hayes, 2018). Demographics (age, gender, parents' highest education level) were also included as covariates in the model. Statistically significant interactions were probed by plotting behaviour and trait self-control or implicit self-control at three conditional values of beliefs in self-control: the mean, and one standard deviation (SD) above and below the mean. Conditional moderation plots were implemented using the ggplot2 package in $\mathrm{R}$ ( $\mathrm{R}$ Development Core Team, 2017). ${ }^{4}$

\footnotetext{
${ }^{2}$ We opted not to include the non-planning scale from the Barratt impulsiveness scale in our analysis due to substantive conceptual and empirical overlap between this construct and trait self-control, and the need to avoid redundancy and potential problems with collinearity.

${ }^{3}$ We re-ran our analyses retaining the three excluded cases and imputed missing using the full information maximum likelihood method available in the lavaan package. Results were virtually identical to the non-imputed model, so we report the analysis omitting the four cases with missing data. Model results including all cases with imputed data are available online: https://osf.io/ ywrzc

${ }^{4}$ Analysis scripts for the moderated regression analyses are available online: https://osf.io/ywrzc
} 


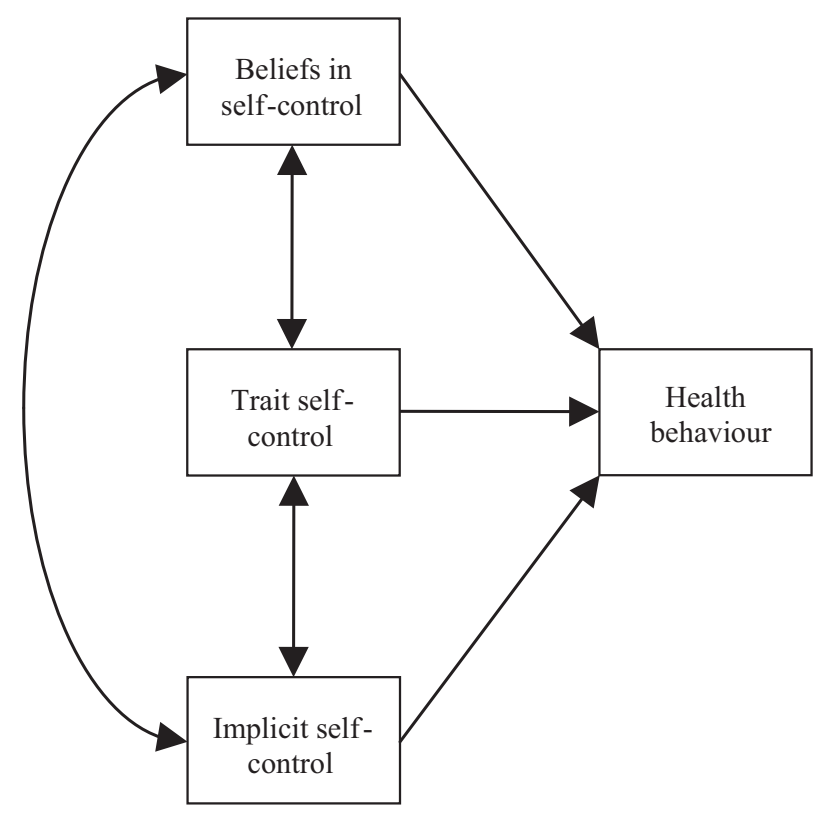

Figure 2. Path model depicting simultaneous regression of health behaviour on implicit self-control, trait self-control, and beliefs in self-control (Model 2).

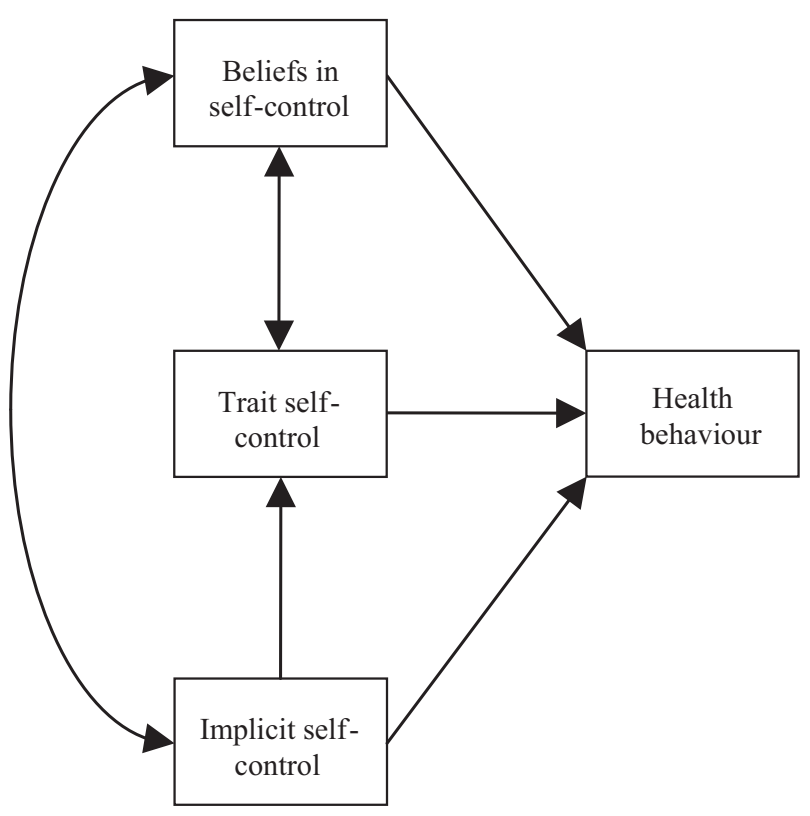

Figure 3. Path model depicting simultaneous regression of health behaviour on implicit self-control, trait self-control, and beliefs in self-control including the indirect effect of implicit self-control on health behaviour through trait self-control (Model 3). 


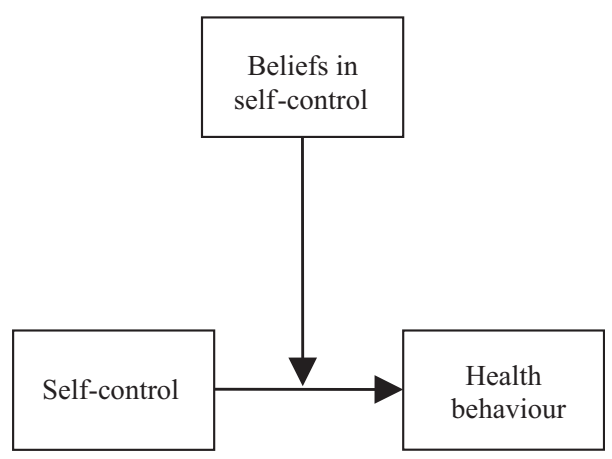

Figure 4. Path model depicting interactive effect of self-control and self-control beliefs on health behaviour (Model 4). Moderation of the relationship between trait self-control and health behaviour, and between implicit self-control and health behaviour, by beliefs in self-control was tested in separate models.

\section{Results}

\section{Correlation analyses}

Three participants were missing IAT data, and one participant did not report their alcohol consumption in week 1 , so data from four participants were excluded from the final analysis leaving a final sample of $172^{5}$. Descriptive statistics, reliabilities coefficients, and correlations among study variables are presented in Table 1 . Reliability coefficients exceeded cut-off values for acceptable internal consistency, with the exception of the alpha coefficient for the newly developed implicit self-control IAT $(\alpha=.551)$. However, correlations of the mean latencies for the initial target concept discrimination and the reverse target concept discrimination $(r=.665, p<.001)$ blocks of trials, and between the initial target concept discrimination and the associated attribute discrimination $(r=.613, p<.001)$ blocks of trials, were large and statistically significant and indicative of internally consistent responses for the IAT. In addition, implicit self-control correlated negatively with the non-planning scale of the Barratt impulsiveness scale and positively with trait self-control. This pattern of relations was congruent with our predictions and is consistent with previous research examining relations between measures of implicit constructs and explicit measures of the same construct, and measures of conceptually related constructs (Gawronski \& Brannon, 2019; Nosek, Greenwald, \& Banaji, 2005). Implicit self-control was also negatively correlated with the impulsive eating and drinking, exercise avoidance, and alcohol consumption, and positively correlated with physical activity, although sizes of the relations were small and the coefficients for alcohol and exercise avoidance fell short of conventional statistical significance by a trivial margin ( $p \mathrm{~s}$ $<$.079). These patterns of relations are consistent with the expectation that self-control dispositions that are not accessible to the individual exhibit relationships with multiple behaviours. Taken together, these findings provide preliminary evidence for the concurrent validity of the implicit self-control measure. Furthermore, trait self-control and self-control beliefs were positively correlated, and all three self-control constructs were negatively related to the non-planning impulsivity measure. The pattern of

\footnotetext{
${ }^{5}$ We re-ran our analyses retaining the four excluded cases and imputed missing using the full information maximum likelihood method available in the lavaan package. Results were virtually identical to the non-imputed model, so we retained that analysis.
} 


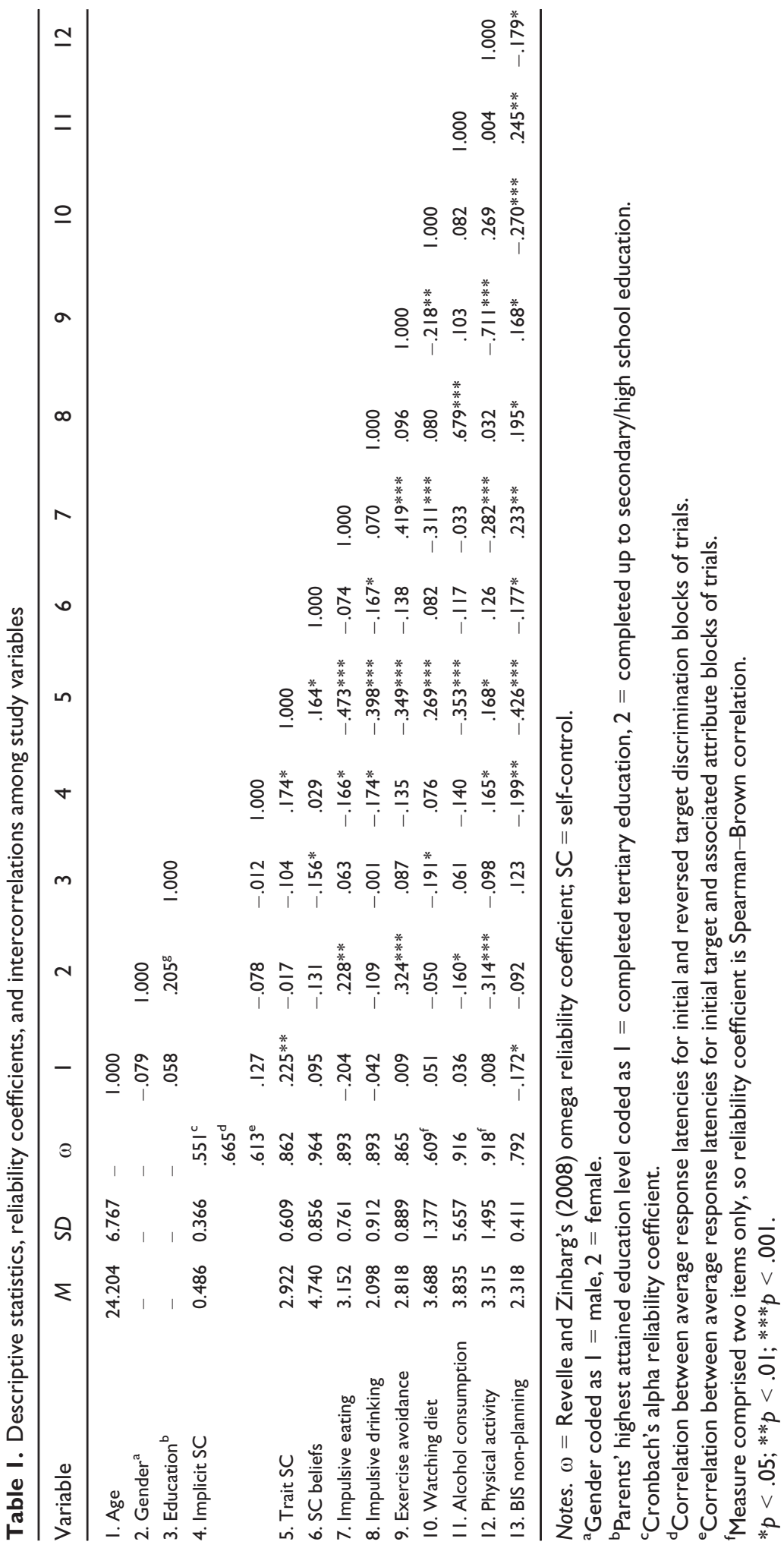


I2 Martin S. Hagger et al.

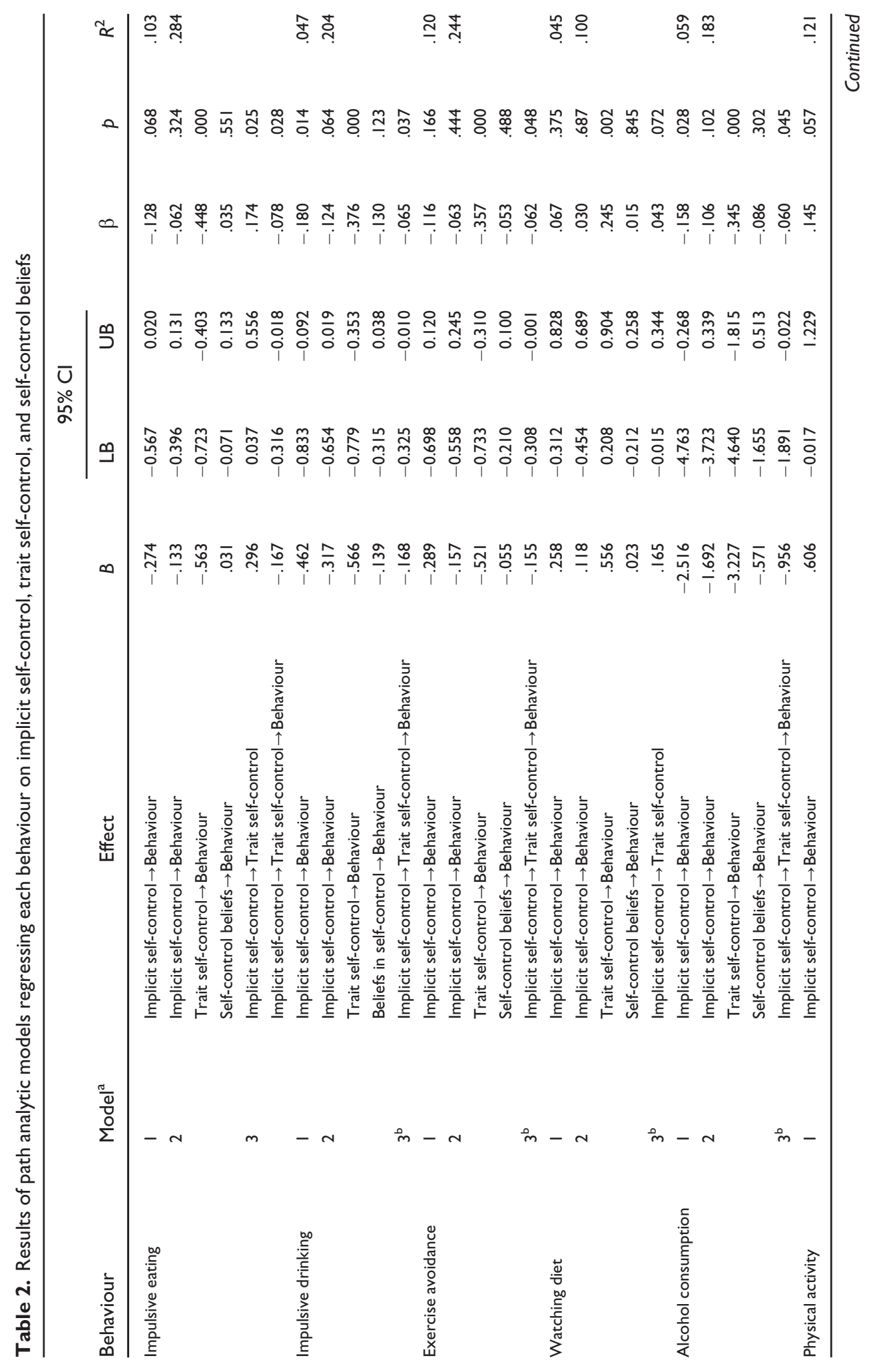




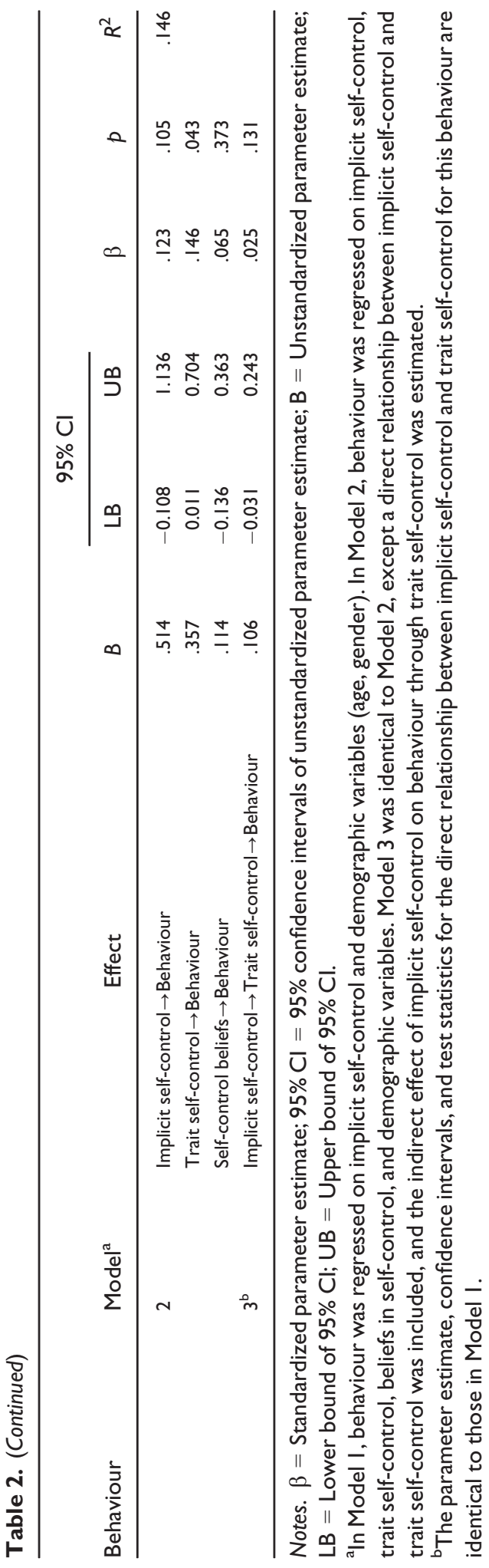


correlations among the self-control constructs provides evidence for the convergent validity of the explicit and implicit measures of self-control.

\section{Path analyses}

Parameter estimates and confidence intervals for the hypothesized models are presented in Table $2^{6}$. Model 1 tested relations between implicit self-control and health-related behaviours controlling for demographic variables only. Results indicated statistically significant negative relations between implicit self-control and impulsive alcohol drinking and alcohol consumption. In addition, relations between implicit self-control and exercise behaviour and impulsive eating were short of the conventional level of statistical significance by a trivial margin. The size of these relations was small-to-medium in all cases with standardized $\beta$ 's $\leq .180$ (Funder \& Ozer, 2019). Including trait self-control and beliefs about self-control as additional predictors of the behavioural variables in Model 2 revealed statistically significant negative relationships between trait self-control and impulsive eating, impulsive drinking, exercise avoidance, and alcohol consumption, and positive relationships between trait self-control and watching diet and physical activity participation. In contrast, there were no relationships between self-control beliefs and any of the behavioural outcomes. Furthermore, relations between implicit self-control and behavioural outcomes were attenuated compared to Model 1. In Model 3, we included a direct relationship between implicit self-control and trait self-control, and an indirect effect of implicit self-control on the behavioural outcomes through trait self-control, to establish the extent to which trait self-control subsumed relations between implicit selfcontrol and behavioural outcomes. Results indicated a statistically significant direct relationship between implicit self-control and trait self-control, consistent with the zeroorder correlation between these two constructs. We also found negative indirect effects of implicit self-control on impulsive eating, impulsive drinking, exercise avoidance, and alcohol consumption through trait self-control. As before, however, sizes of the indirect effects were small (upsilon range $=.001$ to .006 ; Lachowicz et al., 2018).

\section{Moderation analyses}

We tested whether self-control beliefs moderated relations between trait self-control and implicit self-control on behavioural outcomes (Model 4) ${ }^{7}$. Results revealed statistically significant interaction between trait self-control and self-control beliefs in the prediction of impulsive drinking $(\beta=.207, p=.010)$, and implicit self-control and self-control beliefs on exercise avoidance $(\beta=.212, p=.015)$ and physical activity participation $(\beta=.197, p=.005)$. Probing the interactions, we found larger negative relations between trait self-control and impulsive drinking for values of self-control beliefs at $1 S D$ below the mean $(\beta=-.584, p<.001)$, compared to relations at $(\beta=-.378, p<.001)$, and $1 S D$ above $(\beta=-.171, p<.001)$ the mean. We also found larger negative relations between implicit self-control and exercise avoidance for values of self-control beliefs at 1 $S D$ below $(\beta=-.344, p=.005)$, compared to relations at $(\beta=-.132, p=.098)$, and 1 $S D$ above $(\beta=.079, p=.485)$ the mean. In addition, we found larger relations between implicit self-control and physical activity for values of self-control beliefs at $1 S D$ below the

\footnotetext{
${ }^{6}$ Full results of path analytic models are presented in Appendix S4.

${ }^{7}$ Full results of moderated path analytic models are presented in Appendix S5.
} 
mean $(\beta=.361, p<.001)$, relative to values at $(\beta=.164, p=.028)$, and $1 S D$ above $(\beta=-.033, p=.707)$ the mean ${ }^{8}$.

\section{Discussion}

We tested the independent contribution of implicit self-control, trait self-control, and selfcontrol beliefs in the prediction of health-related behaviours. We also tested the moderating effect of self-control beliefs on relations between of trait self-control and health behaviours, and between implicit self-control and health behaviours. Correlation analyses indicated statistically significant positive relations among the three self-control constructs, as predicted. We found significant negative relations between implicit selfcontrol and impulsive drinking and alcohol consumption. We also found a negative relationship between implicit self-control and impulsive eating, and a positive relationship between implicit self-control and physical activity participation, both of which failed to reach the conventional significance level by a trivial margin. In contrast to predictions, relations between implicit self-control and other behaviours were non-significant. Including trait self-control and self-control beliefs as predictors of behavioural outcomes alongside implicit self-control revealed significant relations between trait self-control and all behaviours, although sizes of the relations varied with the smallest for physical activity. There were no relations between self-control beliefs and behavioural outcomes. Analysis of indirect effects in models including direct relations between implicit self-control and behaviour, and between trait self-control and behaviour, revealed significant indirect effects of implicit self-control on impulsive eating and drinking, exercise avoidance, and alcohol consumption through trait self-control. Finally, self-control beliefs moderated relations between trait self-control and impulsive drinking, and moderated relations between implicit self-control and exercise avoidance and physical activity participation.

Overall, we did not find strong evidence to support our prediction that implicit selfcontrol would predict health-related behaviours. Our predictions were only supported for the relationship between implicit self-control and alcohol-related behaviours with small effect sizes, and for impulsive eating and physical activity participation, but the coefficients for the latter two relationships approached, but did not meet, conventional levels of statistical significance. One possible interpretation of a direct relation between implicit self-control and behaviour, and therefore a more implicit process by which trait self-control relates to behaviour, is that individuals have behavioural scripts or schema that become active when relevant cues to behavioural alternatives that might derail a target goal-directed behaviour are presented (Abelson, 1981; Schnabel, Asendorpf, \& Greenwald, 2008). The scripts are motor patterns stored in memory that when activated assist the individual in inhibiting the derailing cues and attending to cues to regulate the target behaviour. The scripts may have been developed through experience in managing derailing cues over time and may have once been serviced by more deliberative, effortful processes. However, current data seem to indicate that implicit orientations only make a modest contribution to explaining variance in managing health-related behaviours. These results raise questions over whether individuals' engagement in these behaviours occurs as a result of script-based decisions that affect behaviour beyond an individual's awareness.

\footnotetext{
${ }^{8}$ Plots of the relationship between implicit self-control and behaviour, and between trait self-control and behaviour, at each level of self-control beliefs are available online from the Open Science Framework project for this article: https:/losf.io/ywrzcl
} 
Far more pervasive in the current study were the relations between trait self-control and health behaviours. Trait self-control was a predictor of all behaviours in the current model, with a predictable pattern: negative relations between trait self-control and impulsive eating and drinking, exercise avoidance, and alcohol consumption, and positive relations between trait self-control and watching diet and physical activity participation. These results are consistent with the large body of research demonstrating consistent small-to-medium-sized relations between trait self-control and health behaviours (e.g., Hamilton, Fleig, \& Hagger, 2019; Hankonen et al., 2014; Junger \& van Kampen, 2010; Tangney et al., 2004). Furthermore, it seems that any relations between implicit selfcontrol and the current set of health behaviours were attenuated when trait self-control was included as a preductor. Given that trait self-control and implicit self-control were significantly and positively correlated, current findings likely indicate that any variance shared between the health behaviours and implicit self-control was also shared by trait self-control.

From a theoretical perspective, these results provide preliminary evidence that trait self-control is sufficient in capturing implicit self-control, and implicit self-control does not add to explaining variance in behaviour. Our findings contrast with a previous studies that have examined relations between measures of implicit self-control and health behaviour independent of trait measures (Churchill \& Jessop, 2011; Huntjens, Rijkeboer, Krakau, \& de Jong, 2014). For example, Churchill and Jessop (2011) demonstrated that implicit selfcontrol was related to unhealthy snacking and that the effects were independent of measures of impulsivity, while Huntjens et al. (2014) found that an implicit measure was related, albeit weakly, with laboratory-based measures of impulsive behaviour. However, methods for both studies differed from the present study. Churchill and Jessop used a measure of implicit self-control that was specific to snacking behaviours and involved approach and avoidance responses to healthy and unhealthy food-related stimulus words, and did not include trait measures. Huntjens et al. used a measure of measure of the obsessive and compulsive self, which focused on 'chaotic' and 'orderly' primes, and did not capture the essence of self-control as a construct. These methodological variations preclude direct comparisons with the current study. A strength of our study is that we examined relations between implicit self-control and multiple behaviours, and also included concurrent measures of trait self-control, which allowed us to test the generalizability of relations across behaviours. Our results cast doubt on the viability of implicit measures of self-control in accounting for unique variance in health-related behaviours when accounting for trait self-control.

Knowledge that trait self-control is associated with participation in health-related behaviours also has practical value. Current findings and previous evidence suggest that deficits in self-control may leave individuals vulnerable to conditions and cues that may initiate enactment of unhealthy behaviours such as impulsive eating or drinking, or impulse-related alternative health-related behaviours such as opting for sedentary activities instead of physical activity participation (e.g., Baldwin et al., 2018). As with all dispositional constructs that predict behavioural outcomes, a concern is that because traits are relatively stable and unmalleable, interventions designed to improve dispositional self-control may not be successful in bringing about necessary behaviour change. However, knowledge of potential deficits attributable to low trait self-control suggests that prudent management of the presentation of conditions that may cue up unhealthy behaviours, or initiate healthy ones, may be a useful avenue for interventions. Strategies such as providing individuals with the capability to monitor cues and behavioural skills like contingency planning, environmental restructuring, and nudging may be effective in 
assisting people to overcome their specific vulnerabilities afforded by low trait self-control (Hagger, 2019; Harkin et al., 2016; Hollands et al., 2017; Nurmi, Hagger, Haukkala, Araújo-Soares, \& Hankonen, 2016; Verhoeven, Adriaanse, de Vet, Fennis, \& de Ridder, 2014).

The potential of these management strategies notwithstanding, there is research suggesting that interventions aimed at promoting greater trait-level self-control may also be effective. One avenue of research is impulse control training. Several meta-analytic studies have demonstrated that individuals trained to resist acting impulsively resulted in increased participation in health behaviours, with small-to-medium-sized effects (Allom, Mullan, \& Hagger, 2016; Friese, Frankenbach, Job, \& Loschelder, 2016; Jones et al., 2016). Training self-control capacity may, therefore, be a promising avenue for interventions aimed at promoting better inhibitory control, a hallmark of individuals high in trait selfcontrol. Such interventions are predicated on formative evidence that trait self-control is consistently related to such behaviours. However, there is the suggestion that a mechanism by which self-control training acts in changing behaviour is by altering their beliefs about their self-control capacity (Walton \& Wilson, 2018).

We did not find consistent moderation of the relationship between trait self-control and health-related behaviours, and between implicit self-control and health-related behaviours, by self-control beliefs. The most consistent findings were for exercise avoidance and physical activity participation: for both behaviours, we found larger relations between implicit self-control and behaviour when individuals endorsed a limited perspective of self-control. We also found that self-control beliefs moderated the relationship between trait self-control and impulsive drinking, which followed the same pattern. These findings provide preliminary evidence, at least for some behaviours, that beliefs in self-control affect the relationship between dispositional self-control and health behaviour. A possible reason for this pattern of relations is that individuals who believe that self-control is limited are more likely to be affected by relatively stable, unchanging self-control constructs, captured by trait self-control and implicit self-control. For example, among individuals endorsing the belief that self-control is limited, those with low dispositional self-control are more likely to avoid exercise and drink impulsively compared to those with high dispositional self-control. This pattern of relations for dispositional self-control on behaviours was also true for individuals endorsing nonlimited beliefs in self-control, but the relative magnitude of the relationships was smaller. To speculate, this finding opens up the possibility that behaviours for those endorsing non-limited beliefs in self-control are more likely to be determined by motivational factors that vary with situation and context, and less likely to be affected by dispositional factors. In the present study, we could not unequivocally verify this explanation for these behaviours as we did not measure motivational factors. We look to future research that measures trait and implicit self-control constructs and self-control beliefs in conjunction with measures of motivational factors like intentions, attitudes, and self-efficacy.

\section{Strengths and limitations}

The current research has numerous strengths including the development of a measure of implicit self-control from first principles, and testing the unique contribution of implicit self-control to the prediction of multiple health behaviours alongside measures of trait selfcontrol and beliefs in self-control. We also tested the important mechanistic role of selfcontrol beliefs as a moderator of the relationship between self-control and health-related behaviour. However, several limitations of the study should be considered when 
interpreting current findings. First, although we provided preliminary evidence in support of the concurrent and predictive validity of our new measure of implicit selfcontrol alongside measures of conceptually related constructs (trait self-control, the nonplanning subscale of impulsivity, and beliefs in self-control), and measures of behaviour, the reliability coefficient for the measure fell below commonly accepted cut-off values. Future research should seek to provide further tests of internal consistency of the implicit self-control measure, as well as additional tests of concurrent and predictive validity against conceptually related measures, in multiple behaviours, populations, and contexts. For example, examining relationships between the implicit self-control measures and multiple behavioural measures of self-control, consistent with similar tests conducted for trait self-control measures (e.g., Allom, Panetta, Mullan, \& Hagger, 2016; Hagger et al., 2013), would be a useful avenue for future research.

In addition, we have suggested that the measure of implicit self-control is closely aligned, conceptually, with trait self-control. However, the relatively small size of the correlation between the implicit measure and trait self-control $(r=.174, p<.001)$ suggests the degree of overlap is relatively modest. It is, therefore, possible that the implicit measure may reflect other aspects of self-control and may diverge from trait selfcontrol in the prediction of behavioural outcomes depending on circumstance. For example, the implicit measure uses self versus other as the attribute category, so may reflect self-identity as a 'self-controlled person', or a descriptive norm reflecting individuals' characterizing themselves as a member of groups of people with good selfcontrol. Examination of associations between the implicit measure and measures of selfcontrol self-identity and descriptive norms would shed light on this speculative premise. It would also be interesting to examine the sensitivity of the implicit measure to change, through, for example, self-control training or activating a self-control self-identity or descriptive norm. Testing such sensitivity may provide information on whether implicit self-control is stable and trait-like, and, therefore, similar to trait self-control, or changeable and state-like, consistent with a belief-based perspective of self-control as a self-identity or descriptive norm.

Second, the present study adopted a correlational, cross-sectional design. Any directional or causal relations are, therefore, inferred from theory not the data. Research adopting prospective and experimental designs is needed to evaluate how the current self-control constructs may explain change in variables over time. In particular, proper inference of causal relations between constructs should model change in both the predictor or independent variable and the outcome or dependent variable. In the context of the present study, this might mean testing the effect of an experimental manipulation or intervention aimed at changing implicit self-control on changes in behaviour before and after the manipulation or intervention, against a control or 'usual care' comparison group.

Third, we relied entirely on self-report measures of behaviour. Although there is demonstrable evidence to support the concurrent validity of self-report measures of behaviour alongside more objective measures (e.g., Cust et al., 2008; Simons, Wills, Emery, \& Marks, 2015), they are, nevertheless, subject to bias including socially desirable responses and reporting bias due to, for example, recall failure (DuRant \& Ainsworth, 1996). In addition, exclusive use of self-reports for both psychological and behavioural measures has the potential to introduce common method variance (Hagger, Chatzisarantis, Culverhouse, \& Biddle, 2003). Taken together, these biases may have influenced the relationships among the constructs and behavioural measures in the current study. It should be noted, however, that previous meta-analytic research has demonstrated no variability in the size of the relationship between trait self-control and behavioural 
outcomes in groups of studies using self-report measures of behaviour and studies measuring behaviour by objective means (de Ridder et al., 2012). Nevertheless, future research should seek to verify the patterns of relations tested in the current study using objective measures of behaviour such as physical activity measured using accelerometry, and alcohol and dietary intake verified through observation or weighing.

Fourth, current results should be replicated in non-student samples. Although research on students may be informative of potential mechanisms, there may be characteristics of this homogenous group that have potential to affect findings, and replication in samples more representative of the general population is needed.

Fifth, we did not include a measure of intentions or motivation. Inclusion of such measures, particularly those that are time-lagged, may provide important information on the processes by which the self-control constructs identified in the current study relate to behaviour. In addition, inclusion of a measure of goal progress may shed light on the extent to which self-control afforded sustained focus on goal-directed behaviour.

Finally, our moderator analyses suggested that relations between dispositional selfcontrol, represented by the trait self-control and implicit self-control constructs, and behaviour were less relevant when individuals endorsed a non-limited perspective on selfcontrol. We speculated that motivational factors such as intentions, attitudes, and selfefficacy were more relevant for these individuals. The absence of such measures is a limitation of the current research; thus, future research that includes these motivational factors alongside trait self-control, implicit self-control, and beliefs in self-control is needed.

\section{Conclusion}

The present study set out to examine the unique contribution of implicit self-control, trait self-control, and self-control beliefs in the prediction of health-related behaviour. Findings indicate that although implicit self-control predicted some of the behaviours, the size of the relations was small by comparison. Current findings did, however, provide support for consistent relations between trait self-control and health-related behaviours. Any relationship between implicit self-control and behaviour seemed to be subsumed by trait self-control. In addition, lay beliefs in self-control did not predict behaviours, but moderated relations between trait and implicit self-control and behaviour for some behaviours. Our findings suggest that researchers interested in self-control on health behaviours should focus on relations between trait self-control and behaviour, and the potential moderating effect of self-control beliefs. Current results add to the expanding literature on relations between trait self-control and behaviour and point to the potential for interventions aimed at managing self-control deficits such as self-monitoring (Harkin et al., 2016), cue monitoring (Verhoeven et al., 2014), and environmental restructuring or nudging (Hagger, 2019), or boosting self-control capacity such as self-control training (Friese et al., 2016) to promoting health behaviour change.

\section{Acknowledgements}

Martin S. Hagger's contribution was supported by a Finland Distinguished Professor (FiDiPro) award (Dnro 1801/31/2105) from Business Finland and a Visiting Professorship from the Université Paris Nanterre. Results reported in this article were presented as part of an invited lecture by Martin S. Hagger at the 2018 Dresden Symposium on "Volition and Self-Control: From Metaphors to Mechanisms", Dresden, Germany, August 29-31, 2018. Daniel F. Gucciardi 
was supported by a Curtin Research Fellowship. All procedures performed in studies involving human participants were in accordance with the ethical standards of the institutional and/or national research committee and with the 1964 Helsinki declaration and its later amendments or comparable ethical standards. Informed consent was obtained from all individual participants included in the study.

\section{Conflict of interest}

All authors declare no conflict of interest.

\section{References}

Abelson, R. P. (1981). Psychological status of the script concept. American Psychologist, 36, $715-$ 729. https://doi.org/10.1037/0003-066X.36.7.715

Allom, V., Mullan, B. A., \& Hagger, M. S. (2016). Does inhibitory control training improve health behaviour? A meta-analysis. Health Psychology Review, 10, 168-186. https://doi.org/10.1080/ 17437199.2015 .1051078

Allom, V., Mullan, B. A., Monds, L., Orbell, S., Hamilton, K., Rebar, A. L., \& Hagger, M. S. (2018). Reflective and impulsive processes underlying saving behavior and the additional roles of selfcontrol and habit. Journal of Neuroscience, Psychology, and Economics, 1(3), 135-146.

Allom, V., Panetta, G., Mullan, B., \& Hagger, M. S. (2016). Self-report and behavioural approaches to the measurement of self-control: Are we assessing the same construct? Personality and Individual Differences, 90, 137-142. https://doi.org/10.1016/j.paid.2015.10.051

Baldwin, C. L., Finley, A. J., Garrison, K. E., Crowell, A. L., \& Schmeichel, B. J. (2018). Higher trait selfcontrol is associated with less intense visceral states. Self and Identity. Advance online publication. https://doi.org/10.1080/15298868.2018.1495666

Baumeister, R. F., \& Heatherton, T. F. (1996). Self-regulation failure: An overview. Psychological Inquiry, 7, 1-15. https://doi.org/10.1207/s15327965pli0701_1

Bernecker, K., \& Job, V. (2015). Beliefs about willpower moderate the effect of previous day demands on next day's expectations and effective goal striving. Frontiers in Psychology, 6, 1496. https://doi.org/10.3389/fpsyg.2015.01496

Caudwell, K. M., \& Hagger, M. S. (2015). Predicting alcohol pre-drinking in Australian undergraduate students using an integrated theoretical model. Applied Psychology: Health and Well-Being, 7 , 188-213. https://doi.org/10.1111/aphw.12044

Churchill, S., \& Jessop, D. C. (2011). Reflective and non-reflective antecedents of health-related behaviour: Exploring the relative contributions of impulsivity and implicit self-control to the prediction of dietary behaviour. British Journal of Health Psychology, 16, 257-272. https://doi. org/10.1348/135910710x498688

Conner, M. T., \& Abraham, C. (2001). Conscientiousness and the theory of planned behavior: Toward a more complete model of the antecedents of intentions and behavior. Personality and Social Psychology Bulletin, 27, 1547-1561. https://doi.org/10.1177/01461672012711014

Courneya, K. S., Bobick, T. M., \& Schinke, R. J. (1999). Does the theory of planned behavior mediate the relationship between personality and exercise behavior. Basic and Applied Social Psychology, 21, 317-324. https://doi.org/10.1207/S15324834BASP2104_5

Cust, A., Smith, B., Chau, J., van der Ploeg, H., Friedenreich, C., Armstrong, B., \& Bauman, A. (2008). Validity and repeatability of the EPIC physical activity questionnaire: A validation study using accelerometers as an objective measure. International Journal of Behavioral Nutrition and Physical Activity, 5, 33. https://doi.org/10.1186/1479-5868-5-33

De Ridder, D. T. D., \& De Wit, J. B. F. (2006). Self-regulation in health behaviour: Concepts, theories, and central issues. In D. T. D. De Ridder \& J. B. F. De Wit (Eds.), Self-regulation in bealth behavior (pp. 1-24). Chichester, UK: Wiley. https://doi.org/10.1002/9780470713150.ch1 
de Ridder, D. T. D., Lensvelt-Mulders, G., Finkenauer, C., Stok, F. M., \& Baumeister, R. F. (2012). Taking stock of self-control: A meta-analysis of how trait self-control relates to a wide range of behaviors. Personality and Social Psychology Review, 16, 76-99. https://doi.org/10.1177/ 1088868311418749

Duckworth, A. L., \& Gross, J. J. (2014). Self-control and grit: Related but separable determinants of success. Current Directions in Psychological Science, 23, 319-325. https://doi.org/10.1177/ 0963721414541462

DuRant, R. H., \& Ainsworth, B. E. (1996). The recall of physical activity: Using a cognitive model of the question-answering process. Medicine \& Science in Sports \& Exercise, 28, 1282-1291. https://doi.org/10.1097/00005768-199610000-00012

Ford, E. S., Zhao, G. X., Tsai, J., \& Li, C. Y. (2011). Low-risk lifestyle behaviors and all-cause mortality: Findings from the national health and nutrition examination survey III mortality study. American Journal of Public Health, 101, 1922-1929. https://doi.org/10.2105/ajph.2011. 300167

Friese, M., Frankenbach, J., Job, V., \& Loschelder, D. (2016). Does self-control training improve selfcontrol? A meta-analysis. Perspectives on Psychological Science, 12, 1077-1099. https://doi. org/10.1177/1745691617697076

Funder, D. C., \& Ozer, D. J. (2019). Evaluating effect size in psychological research: Sense and nonsense. Advances in Methods and Practices in Psychological Science, 2, 156-168. https:// doi.org/10.1177/2515245919847202

Gawronski, B., \& Brannon, S. M. (2019). Implicit attitudes and the implicit-explicit dualism. In D. Albarracín \& B. T. Johnson (Eds.), The handbook of attitudes (2nd ed., Vol. 1, pp. 158-196). New York, NY: Psychology Press.

Gottfredson, M. R., \& Hirschi, T. (1990). A general theory of crime. Stanford, CA: Stanford University Press.

Grasmick, H. G., Tittle, C. R., Bursik, Jr, R. J., \& Arneklev, B. J. (1993). Testing the core empirical implications of Gottfredson and Hirschi's General Theory of Crime. Journal of Research in Crime and Delinquency, 30, 5-29. https://doi.org/10.1177/0022427893030001002

Greenwald, A. G., McGhee, D. E., \& Schwartz, J. L. K. (1998). Measuring individual differences in implicit cognition: The implicit association test. Journal of Personality and Social Psychology, 74, 1464-1480. https://doi.org/10.1037//0022-3514.74.6.1464

Greenwald, A. G., Nosek, B. A., \& Banaji, M. R. (2003). Understanding and using the Implicit Association Test: I. An improved scoring algorithm. Journal of Personality and Social Psychology, 85, 197-216. https://doi.org/10.1037/0022-3514.85.2.197

Hagger, M. S. (2013). The multiple pathways by which self-control predicts behavior. Frontiers in Psychology, 4, 849. https://doi.org/10.3389/fpsyg.2013.00849

Hagger, M. S. (2014). The multiple pathways by which trait self-control predicts health behavior. Annals of Behavioral Medicine, 48, 282-283. https://doi.org/10.1007/s12160-014-9631-x

Hagger, M. S. (2019). Habit and physical activity: Theoretical advances, practical implications, and agenda for future research. Psychology of Sport and Exercise, 42, 118-129. https://doi.org/10. 1016/j.psychsport.2018.12.007

Hagger, M. S., Chatzisarantis, N. L. D., Culverhouse, T., \& Biddle, S. J. H. (2003). The processes by which perceived autonomy support in physical education promotes leisure-time physical activity intentions and behavior: A trans-contextual model. Journal of Educational Psychology, 95, 784-795. https://doi.org/10.1037/0022-0663.95.4.784

Hagger, M. S., Chatzisarantis, N. L. D., \& Harris, J. (2006). From psychological need satisfaction to intentional behavior: Testing a motivational sequence in two behavioral contexts. Personality and Social Psychology Bulletin, 32, 131-138. https://doi.org/10.1177/0146167205279905

Hagger, M. S., Panetta, G., Leung, C.-M., Wong, G. G., Wang, J. C. K., Chan, D. K.-C., , . Chatzisarantis, N. L. D. (2013). Chronic inhibition, self-control and eating behavior: Test of a 'resource depletion' model. PLoS ONE, 8, e76888. https://doi.org/10.1371/journal.pone.0076888

Hagger, M. S., Zhang, C. Q., Kangro, E.-M., Ries, F., Wang, J. C. K., Heritage, B., \& Chan, D. K. C. (2018). Trait self-control and self-discipline: Structure, validity, and invariance across national 
groups. Current Psychology. Advance online publication. https://doi.org/10.1007/s12144-0180021-6

Hamilton, K., Fleig, L., \& Hagger, M. S. (2019). Being active in pregnancy: Theory-based factors associated with physical activity among pregnant women. Women and Health, 59, 213-228. https://doi.org/10.1080/03630242.2018.1452835

Hankonen, N., Kinnunen, M., Absetz, P., \& Jallinoja, P. (2014). Why do people high in self-control eat more healthily? Social cognitions as mediators. Annals of Behavioral Medicine, 47, 242-248. https://doi.org/10.1007/s12160-013-9535-1

Harkin, B., Webb, T. L., Chang, B. P. I., Prestwich, A., Conner, M., Kellar, I., . . Sheeran, P. (2016). Does monitoring goal progress promote goal attainment? A meta-analysis of the experimental evidence. Psychological Bulletin, 142, 198-229. https://doi.org/10.1037/bul0000025

Hayes, A. F. (2018). Introduction to mediation, moderation, and conditional process analysis: $A$ regression-based approach (2nd ed.). New York, NY: Guildford Press.

Hofmann, W., Schmeichel, B. J., \& Baddeley, A. D. (2012). Executive functions and self-regulation. Trends in Cognitive Sciences, 16, 174-180. https://doi.org/10.1016/j.tics.2012.01.006

Hollands, G. J., Bignardi, G., Johnston, M., Kelly, M. P., Ogilvie, D., Petticrew, M., . . Marteau, T. M. (2017). The TIPPME intervention typology for changing environments to change behaviour. Nature Human Behavior, 1, 0140. https://doi.org/10.1038/s41562-017-0140

Huntjens, R. J. C., Rijkeboer, M. M., Krakau, A., \& de Jong, P. J. (2014). Implicit versus explicit measures of self-concept of self-control and their differential predictive power for spontaneous trait-relevant behaviors. Journal of Behavior Therapy and Experimental Psychiatry, 45, 1-7. https://doi.org/10.1016/j.jbtep.2013.07.001

Job, V., Dweck, C. S., \& Walton, G. M. (2010). Ego depletion - is it all in your head? Implicit theories about willpower affect self-regulation. Psychological Science, 21, 1686-1693. https://doi.org/ $10.1177 / 0956797610384745$

Job, V., Walton, G. M., Bernecker, K., \& Dweck, C. S. (2015). Implicit theories about willpower predict self-regulation and grades in everyday life. Journal of Personality and Social Psychology, 108, 637-647. https://doi.org/10.1037/pspp0000014

Jones, A., Di Lemma, L. C. G., Robinson, E., Christiansen, P., Nolan, S., Tudur-Smith, C., \& Field, M. (2016). Inhibitory control training for appetitive behaviour change: A meta-analytic investigation of mechanisms of action and moderators of effectiveness. Appetite, 97, 16-28. https://doi.org/10.1016/j.appet.2015.11.013

Junger, M., \& van Kampen, M. (2010). Cognitive ability and self-control in relation to dietary habits, physical activity and bodyweight in adolescents. International Journal of Behavioral Nutrition and Physical Activity, 7, 22. https://doi.org/10.1186/1479-5868-7-22

Kok, G., Gottlieb, N. H., Peters, G.-J. Y., Mullen, P. D., Parcel, G. S., Ruiter, R. A. C., . . Bartholomew, L. K. (2016). A taxonomy of behavior change methods: An intervention mapping approach. Health Psychology Review, 10, 297-312. https://doi.org/10.1080/17437199.2015.1077155

Lachowicz, M. J., Preacher, K. J., \& Kelley, K. (2018). A novel measure of effect size for mediation analysis. Psychological Methods, 23, 244-261. https://doi.org/10.1037/met0000165

Nosek, B. A., Greenwald, A. G., \& Banaji, M. R. (2005). Understanding and using the Implicit Association Test: II. Method variables and construct validity. Personality and Social Psychology Bulletin, 31, 166-180. https://doi.org/10.1177/0146167204271418

Nurmi, J., Hagger, M. S., Haukkala, A., Araújo-Soares, V., \& Hankonen, N. (2016). Relations between autonomous motivation and leisure-time physical activity participation: The mediating role of self-regulation techniques. Journal of Sport and Exercise Psychology, 38, 128-137. https://doi. org/10.1123/jsep.2015-0222

OSBBR (2016). Strategic plan 2017-2021: Healthier lives through behavioral and social sciences. Bethesda, MD: Office of Behavioral and Social Sciences Research, National Institutes of Health.

Paschke, L. M., Dörfel, D., Steimke, R., Trempler, I., Magrabi, A., Ludwig, V. U., . . Walter, H. (2016). Individual differences in self-reported self-control predict successful emotion regulation. Social Cognitive and Affective Neuroscience, 11, 1193-1204. https://doi.org/10.1093/scan/nsw036 
Patton, J. H., Stanford, M. S., \& Barratt, E. S. (1995). Factor structure of the Barratt impulsiveness scale. Journal of Clinical Psychology, 51, 768-774. https://doi.org/10.1002/1097-4679 (199511)51:6<768:AID-JCLP2270510607>3.0.CO;2-1

R Development Core Team (2017). $R$ : A language and environment for statistical computing. Vienna, Austria: R Foundation for Statistical Computing.

Revelle, W., \& Zinbarg, R. E. (2008). Coefficients alpha, beta, omega, and the glb: Comments on sijtsma. Psychometrika, 74, 145. https://doi.org/10.1007/s11336-008-9102-z

Rosseel, Y. (2012). lavaan: An R package for structural equation modeling. Journal of Statistical Software, 48, 1-36. https://doi.org/10.18637/jss.v048.i02

Schnabel, K., Asendorpf, J. B., \& Greenwald, A. G. (2008). Assessment of individual differences in implicit cognition. European Journal of Psychological Assessment, 24, 210-217. https://doi. org/10.1027/1015-5759.24.4.210

Sheeran, P., Klein, W. M. P., \& Rothman, A. J. (2017). Health behavior change: Moving from observation to intervention. Annual Review of Psychology, 68, 573-600. https://doi.org/10. 1146/annurev-psych-010416-044007

Simons, J. S., Wills, T. A., Emery, N. N., \& Marks, R. M. (2015). Quantifying alcohol consumption: Selfreport, transdermal assessment, and prediction of dependence symptoms. Addictive Behaviors, 50, 205-212. https://doi.org/10.1016/j.addbeh.2015.06.042

Tangney, J. P., Baumeister, R. F., \& Boone, A. L. (2004). High self-control predicts good adjustment, less pathology, better grades, and interpersonal success. Journal of Personality, 72, 271-324. https://doi.org/10.1111/j.0022-3506.2004.00263.x

Verhoeven, A. A. C., Adriaanse, M. A., de Vet, E., Fennis, B. M., \& de Ridder, D. T. D. (2014). Identifying the 'if' for 'if-then' plans: Combining implementation intentions with cue-monitoring targeting unhealthy snacking behaviour. Psychology \& Health, 29, 1476-1492. https://doi.org/ 10.1080/08870446.2014.950658

Walton, G. M., \& Wilson, T. D. (2018). Wise interventions: Psychological remedies for social and personal problems. Psychological Review, 125, 617-655. https://doi.org/10.1037/rev0000115

Received 6 September 2018; revised version received 26 March 2019

\section{Supporting Information}

The following supporting information may be found in the online edition of the article:

Appendix S1. Items and response scales for study measures, and information provided for behavioral measures.

Appendix S2. Development of self-control implicit association test.

Appendix S3. Example stimulus trials for the self-control implicit association test. Appendix S4. Full results of path analytic models regressing each behavior on implicit self-control, trait self-control, beliefs in self-control, and demographic variables.

Appendix S5. Results of moderated path analytic models. 